\title{
Rückblick und Danksagung
}

\section{J.-Matthias Graf von der Schulenburg}

Online publiziert: 13. März 2018

(C) Springer-Verlag GmbH Deutschland, ein Teil von Springer Nature 2018

Aus Sicht der Zeitschrift für die gesamte Versicherungswissenschaft ist rückblickend das Jahr 2017 wieder ein erfolgreicher Veröffentlichungsjahrgang gewesen: Band 106 enthält viele interdisziplinäre und aktuelle versicherungswissenschaftliche Veröffentlichungen.

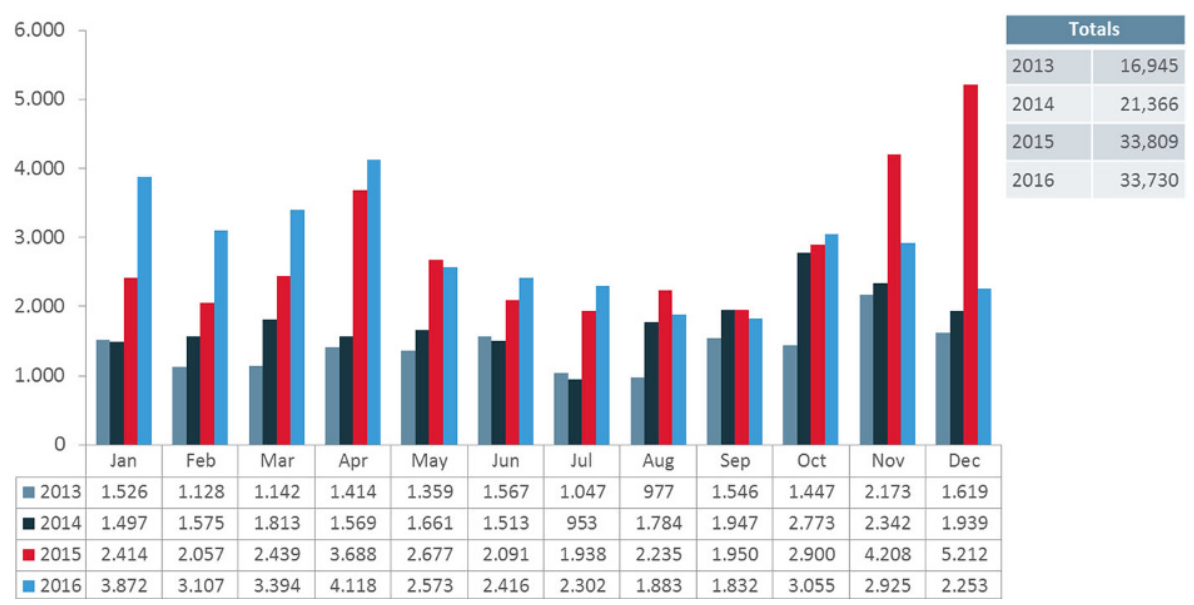

Abb. 1 SpringerLink Full-Text Article Requests 2013-2016. (Source: COUNTER Reporting/Business Warehouse)

J.-M. Graf von der Schulenburg ( $\square)$

Hannover, Deutschland

E-Mail: jms@ivbl.uni-hannover.de 
Die Online-Nachfrage über das Springerportal ist weiterhin auf hohem Niveau für eine interdisziplinäre, in der Regel deutschsprachige Zeitschrift: fast 34.000 Beiträge der Zeitschrift wurden per Download bezogen (siehe Abb. 1).

In 2017 wurde das 500. Reformationsjubiläum gefeiert und auch Martin Luther (1483-1546) hat in einem Zitat die Bedeutung ,guter Literatur“ beschrieben:

Viele Bücher machen nicht gelehrt, viel lesen auch nicht, sondern gute Dinge und oft lesen, wie wenig es auch ist, das macht gelehrt in der Schrift und fromm dazu. (Luther, An den christlichen Adel deutscher Nation, 1520)

Abschließend gebührt mein Dank allen Autoren für ihre Manuskripteinreichungen sowie den unten aufgeführten Bereichsschriftleitern und Gutachtern des letzten Jahres:

- Christian Armbrüster

- Tobias Basse

- Stephan Becher

- Meik Friedrich

- Wolfgang Greiner

- Thomas Hartung

- Mathias Kifmann

- Bernd Lohse

- Ute Lohse

- Angelika May

- Sebastian Reddemann

- Miguel Rodriguez

- Christoph Schwarzbach

- Heinz-Dietrich Steinmeyer

- Manfred Wandt

- Christoph Wegener

- Jan Zeidler 